\title{
Nutrition: what do nurses know and do - a pilot evaluation of nursing staff's knowledge, awareness and practice
}

\author{
S. O’Mahony ${ }^{1}$, J. Hutchinson ${ }^{2}$, A. McConnell ${ }^{2}$, H. Mathieson ${ }^{2}$ and H. McCarthy ${ }^{1}$ \\ ${ }^{1}$ School of Biomedical Sciences, University of Ulster, Coleraine BT52 ISA, UK and ${ }^{2}$ Nutrition and Dietetics Department, \\ Craigavon Area Hospital, BT63 5QQ, UK
}

Nutrition is a core component of quality effective medical care of all patients and as such is the responsibility of all health care professionals $^{(1)}$. The RCN recognises the key role that nurses play in the identification and management of nutritional issues as they have the most frequent contact with patients ${ }^{(2)}$. However, it has been suggested that under-nutrition is frequently unrecognised and one explanation for this has been the lack of nutrition training and subsequent awareness of frontline nursing staff ${ }^{(3)}$.

There were 2 main aims of this study; to quantify the current nutritional knowledge, awareness and practice of adult nursing and nursing support staff, and to investigate the effect of a nutrition education programme on nutrition knowledge awareness and practice among adult nurses and nursing support staff.

Nursing staff ( $n$ 23) completed pre-training questionnaires (28 questions) and from this a Positive Nutrition Education Programme was devised educating nursing staff on nutrition and how to utilise Malnutrition Universal Screening Tool (MUST). A post training questionnaire (25 questions) was then completed one month following training ( $n$ 16). The maximum achievable knowledge score was 31 for both questionnaires. Mean knowledge scores pre and post nutrition education for all staff were not significantly different (21 SD and 23 SD respectively). This may be attributed to the large standard deviations seen or the small sample size. However, for Band 5 nurses (pre-training, $n$ 9; post training, $n$ 6) a significant improvement in mean knowledge scores was notes (22 SD and 27 SD respectively; $P=0.007)$ Nursing staff's nutrition practice, awareness and attitudes to MUST were also analysed.

\begin{tabular}{lcr}
\hline Question & $\begin{array}{c}\text { Nursing staff responding } \\
\text { 'Yes' pre-education }(n \text { 23) }\end{array}$ & $\begin{array}{c}\text { Nursing staff responded } \\
\text { 'Yes' post education }(n \text { 16) }\end{array}$ \\
\hline Practice - Are patients weighed on admission? & $74 \%$ & $94 \%$ \\
Awareness - Is malnutrition a significant problem for NHS today? & $39 \%$ & $82 \%$ \\
MUST - Would MUST be useful on the ward? & $94 \%$ & 0.001 \\
\hline
\end{tabular}

*Values compared using chi-square test, $P<0.05$

This small study highlights the positive attitudes of nursing staff to the use of a nutrition screening tool such as MUST and suggests that a Positive Nutrition Education Programme could improve nutritional knowledge, increase nutritional awareness and result in improved nutritional practices. Further, more detailed studies into the impact of training on nursing nutrition awareness and practice, specifically in relation to MUST may suggest ways to improve compliance with the nutrition screening of patients.

1. NICE (2006) Guideline 32, NICE.

2. Green SM \& Watson R (2007) J Adv Nurs 50, 69-83.

3. Elia M, Zellipour L \& Stratton RJ (2005) Clin Nutr 24, 867-884. 